Supporting Information:

\title{
Isoindigo-based Binary Polymer Blends for Solution- Processing of Semiconducting Nanofiber Networks
}

Aristide Gumyusenge, ${ }^{\dagger}$ Xuyi Luo, ${ }^{\dagger}$ Hongyi Zhang, ${ }^{\dagger}$ Gregory M. Pitch, ${ }^{*}$ Alexander L. Ayzner, ${ }^{*}$ and Jianguo $\mathrm{Mei}^{*},{ }^{\dagger}$

${ }^{\dagger}$ Department of Chemistry, Purdue University, 560 Oval Drive, West Lafayette, Indiana 47907, United States

\$ Department of Physical \& Biological Sciences-Chemistry and Biochemistry, University of California Santa Cruz, 1156 High Street, Santa Cruz, California 95064, United States

Corresponding Author

*jgmei@purdue.edu

Materials Synthesis and Characterization

OFET Devices Fabrication and Characterization

S-4

Glass Transition Temperature (Tg) Measurement and Calculation

S-7

Mechanical Properties Measured by Buckling Method

S-9

Crack-on-Set Strain Analysis and Electrical Measurement

S-11 


\section{Materials Synthesis and Characterization}

P1, P2, P3, and C3 were synthesized as previously reported ${ }^{1-6}$ and characterized by NMR, UVVis absorption, and GPC. The NMR spectrum and the GPC analysis results of the newlyreported C3 are shown in Figure S1 and S2. Polystyrene (Mw 192,000) was purchased from Sigma-Aldrich and used as received. A $10 \mathrm{mg} / \mathrm{mL}$ solution was obtained by stirring overnight in chloroform. Solutions of all the three polymers were obtained in chloroform $(10 \mathrm{mg} / \mathrm{mL})$ and stirred overnight. The corresponding blends were obtained by allowing the respectively vol\% to mix together for at least 1 hour to ensure mixing. Thin films used for morphology studies, GIXRD studies, as well UV-Vis measurements were obtained by spin coating (2000 rpm, $1 \mathrm{~min}$ ) the solutions on cleaned substrates and then annealed to $140^{\circ} \mathrm{C}$ inside the $\mathrm{N}_{2}$ glovebox.

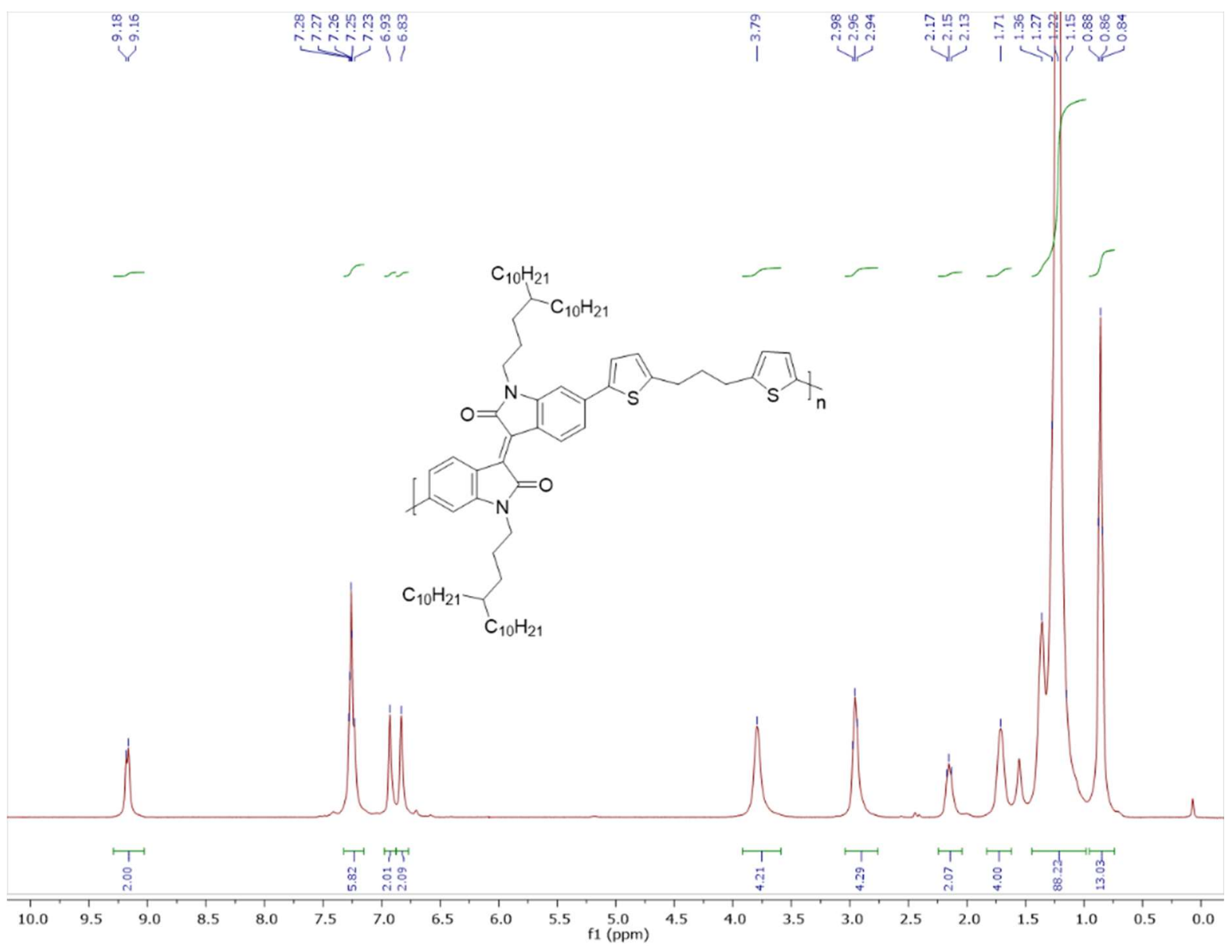

Figure S1. NMR spectra of C3 
Sample :

AG-JM-IIG-C3-121217

Injection Date : $\quad$ 12-Dec-17, 13:54:20

Calibration File : C:IChem3211IDATAICalibrationsI2017April20.CAL

Calibration Date : Monday 01/30/17 16:49:48

Baseline from : $\quad 19.648 \mathrm{~min}$

Mass Distr. from : $19.648 \mathrm{~min}$

MHK - A (Cal.): $\quad 0.000000 E+0$

Eluent : $\quad$ THF

Concentration : $\quad 1.000 \mathrm{~g} / \mathrm{l}$

Detector 1 : RID1A, Refractive Index Signal VA

Baseline to : $\quad 22.915 \mathrm{~min}$ Mass Distr. to : $\quad 22.915 \mathrm{~min}$ MHK - K (Cal.): $\quad 1.000000 E+0 \mathrm{ml} / \mathrm{g}$ Flowrate : Delay volume : $\quad 0.000 \mathrm{ml}$ Acquisition interval : $0.430 \mathrm{sec}$

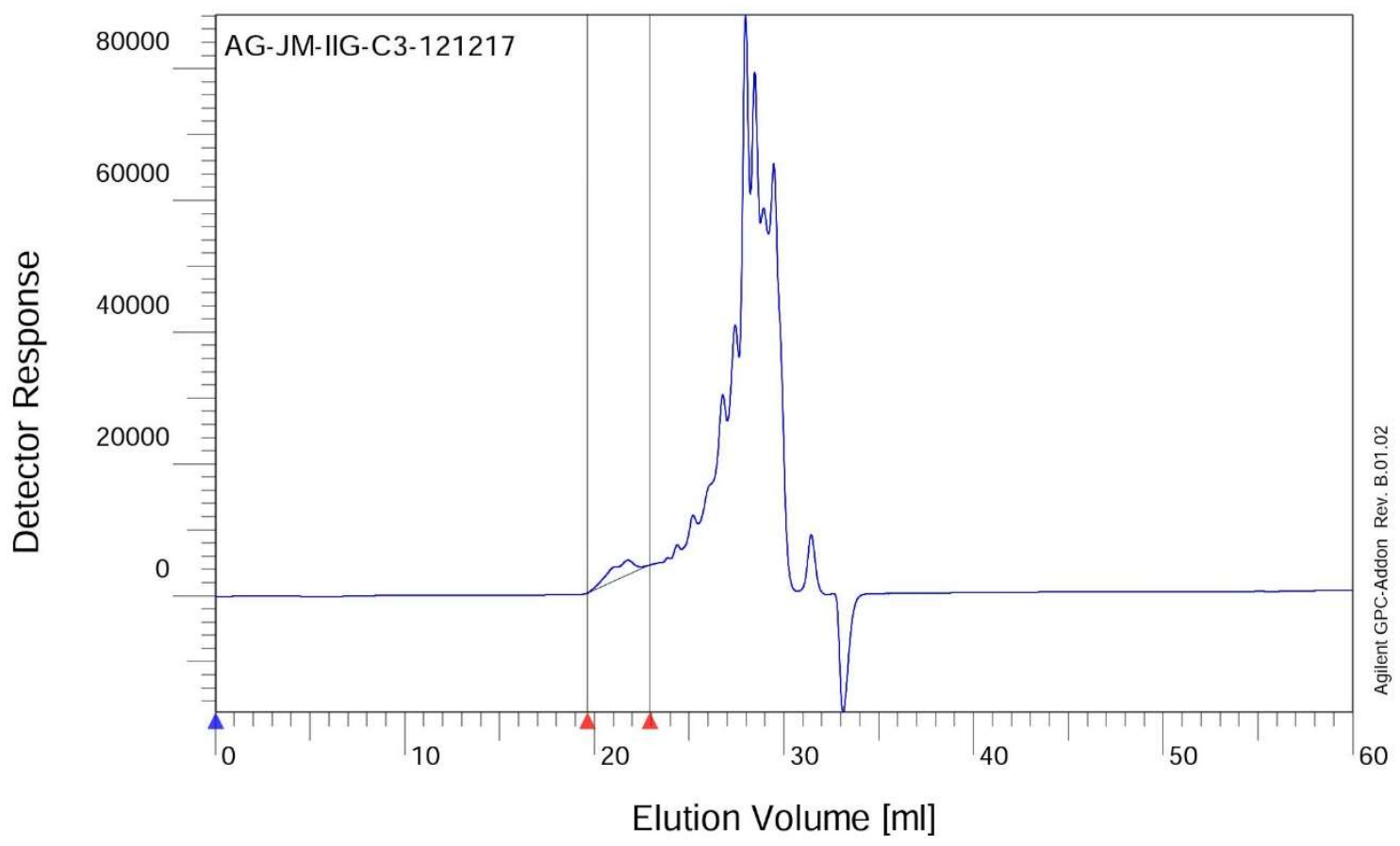

RID1A

$\begin{array}{lll}\overline{M n}: & 2.7292 \mathrm{e} 4 & \mathrm{~g} / \mathrm{mol} \\ \text { Mw : } & 3.2629 \mathrm{e} 4 & \mathrm{~g} / \mathrm{mol} \\ \text { Mz : } & 3.8829 \mathrm{e} 4 & \mathrm{~g} / \mathrm{mol} \\ \text { Mv : } & 0.000000 & \mathrm{~g} / \mathrm{mol} \\ \text { D : } & 1.1956 \mathrm{e} 0 & \\ \text { [n]: } & 0.000000 & \mathrm{ml} / \mathrm{g} \\ \text { Vp : } & 2.1708 \mathrm{e} 1 & \mathrm{ml} \\ \text { Mp : } & 2.2220 \mathrm{e} 4 & \mathrm{~g} / \mathrm{mol} \\ \text { A : } & 3.7367 \mathrm{e} 3 & \mathrm{ml}{ }^{*} \mathrm{~V} \\ \mathbf{1 0 \%} & 1.7424 \mathrm{e} 4 & \mathrm{~g} / \mathrm{mol} \\ \mathbf{3 0 \%} & 2.2846 \mathrm{e} 4 & \mathrm{~g} / \mathrm{mol} \\ \mathbf{5 0 \%} & 2.9630 \mathrm{e} 4 & \mathrm{~g} / \mathrm{mol} \\ \mathbf{7 0} \% & 3.8169 \mathrm{e} 4 & \mathrm{~g} / \mathrm{mol} \\ \mathbf{9 0 \%} & 5.2705 \mathrm{e} 4 & \mathrm{~g} / \mathrm{mol}\end{array}$

Figure S2. Molecular weight analysis of C3 using GPC with tetrahydrofuran as the eluent. 


\section{OFET Devices Fabrication and Characterization}

To fabricate the OFET devices, $\mathrm{Si} / \mathrm{SiO}_{2} / \mathrm{Au}$ substrates were cleaned using piranha mixture (2:1 $18 \mathrm{M} \mathrm{H}_{2} \mathrm{SO}_{4}: 30 \% \mathrm{H}_{2} \mathrm{O}_{2}$ ) for 20 minutes and copious rinsing with water. The cleaned and dried substrates were then modified with a thin layer of octadodecylsilane ${ }^{7}$ in a vacuum oven for 3 hours. The OTS treated surfaces were then rinsed in hexane, chloroform, and then dried with nitrogen air prior to use. The OFET devices were then fabricated by spin casting the $10 \mathrm{mg} / \mathrm{mL}$ solution at ambient. To eliminate any possible thermal history, the thin films were then annealed at $140{ }^{\circ} \mathrm{C}$ inside a $\mathrm{N}_{2}$ glove box for 20 minutes. The films were then allowed to cool to ambient before OFET characterization. Charge carrier mobility measurements were then carried out using Keithley 4200 at ambient. OFET performances were obtained by applying a gate bias from -60 $\mathrm{V}$ to $6 \mathrm{~V}$, with the potential gradient between the source and drain contacts kept at $-60 \mathrm{~V}$. The OFET channel width and length were kept at 1400 and $40 \mu \mathrm{m}$, respectively. The field-effect mobility was calculated in the saturation regime by using the equation $I_{\mathrm{DS}}=\left(\mathrm{WC}_{\mathrm{i}} / 2 \mathrm{~L}\right) \mu\left(V_{\mathrm{G}}-\right.$ $\left.V_{\mathrm{T}}\right)^{2}$, where $I_{\mathrm{DS}}$ is the drain-source current, $\mu$ is the field-effect mobility, $\mathrm{W}$ is the channel width, $\mathrm{L}$ is the channel length, $\mathrm{C}_{\mathrm{i}}$ is the capacitance per unit area of the gate dielectric layer, $V_{\mathrm{G}}$ is the gate voltage, and $V_{\mathrm{T}}$ is the threshold voltage. 

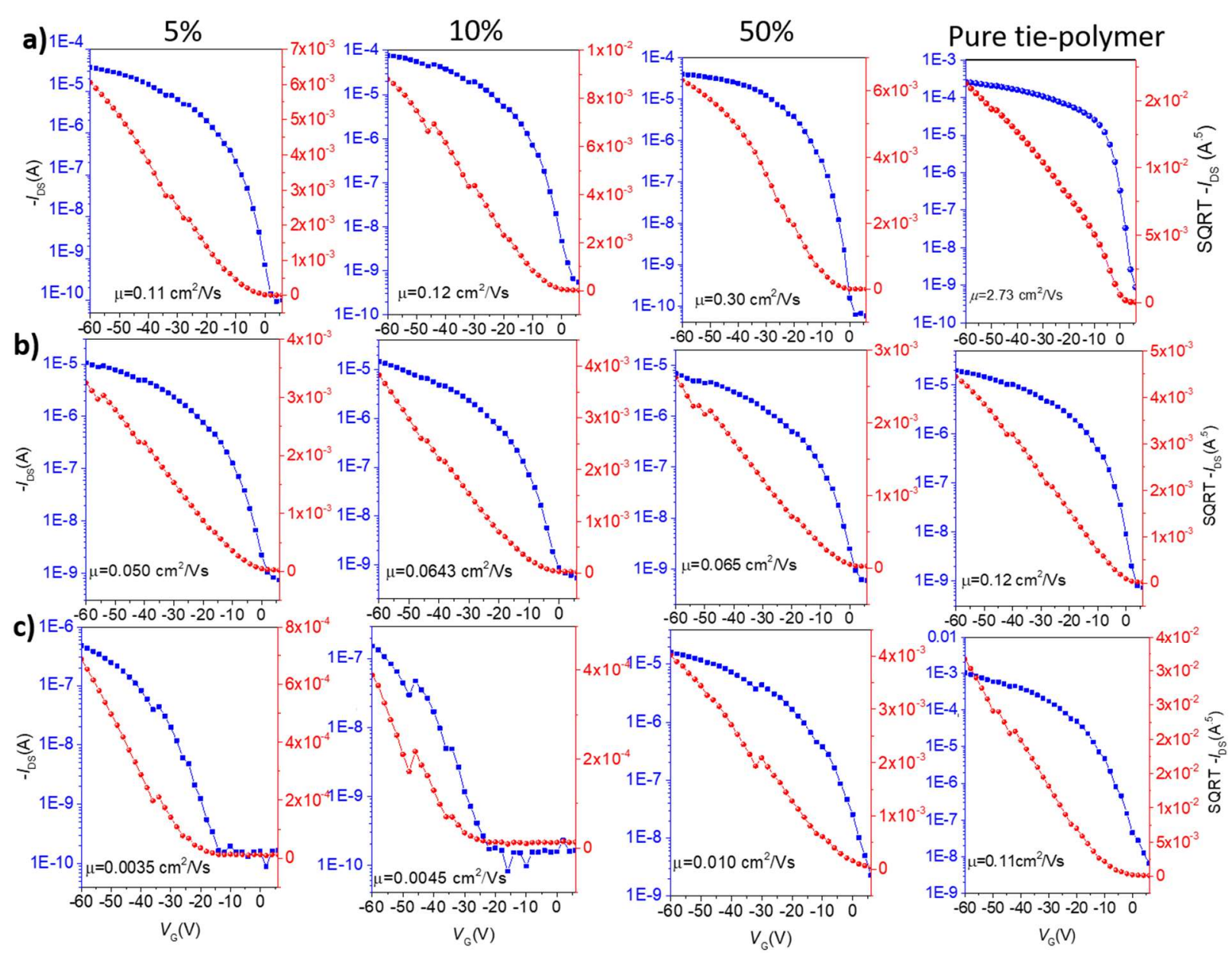

Figure S3. Transfer curves of OFET devices fabricated from representative blend solutions of $\mathrm{C} 3$ in a) P1, b) P2, and c) P3. 

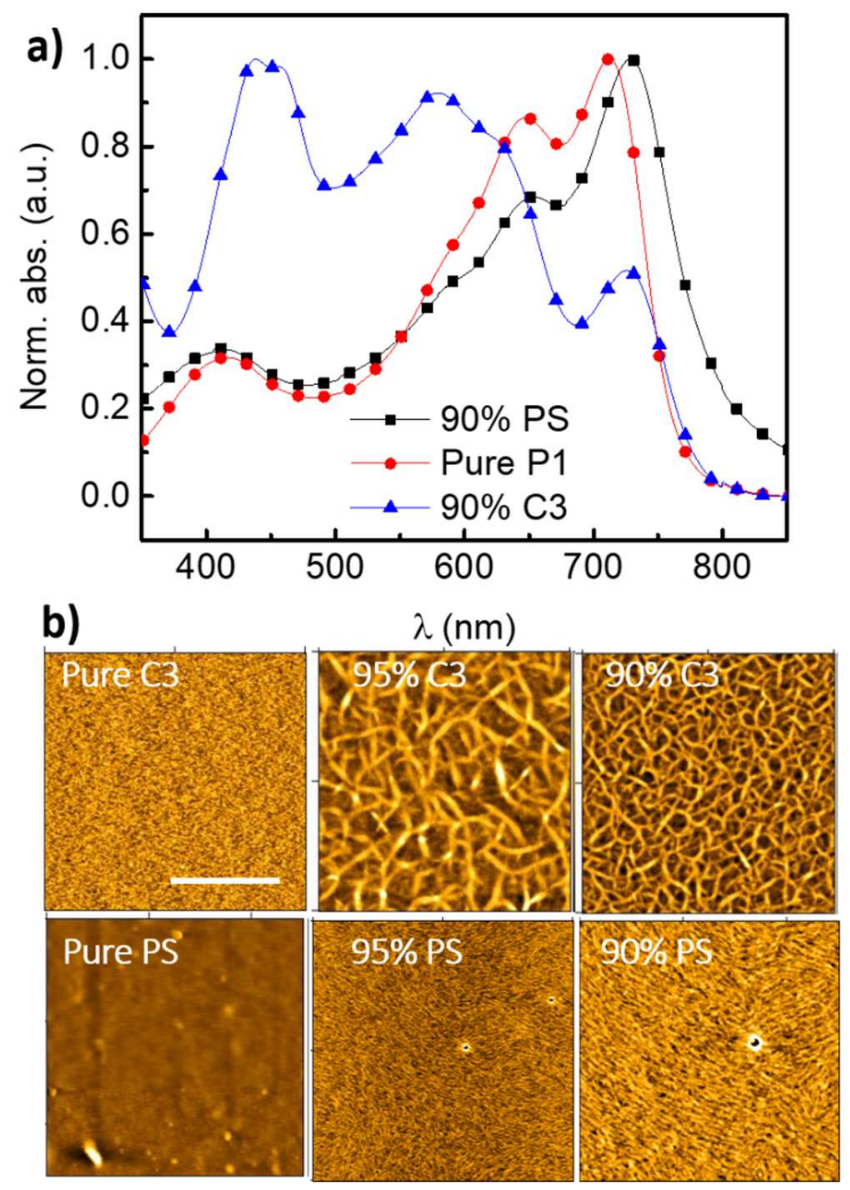

Figure S4. Comparative analysis between designed complementary blends and the common blends using insulating matrices (e.g. polystyrene). a) UV-Vis absorption spectra of thin-films of $\mathrm{P} 1$ blended with the studied matrix, C3, in comparison to polystyrene. The $0-0$ vibronic peak showed a significant red shift only in polystyrene-based blends, while the blends forming nanofibers showed no evidence of long-range aggregation of the tie chains. b) AFM height images of the pure matrices and corresponding blends revealing no formation of fibers when polystyrene is used. 


\section{Glass Transition Temperature (Tg) Measurement and Calculation}

All thin films used for Tg calculations were prepared by spin coating (2000 rpm, 60s) of 10 $\mathrm{mg} / \mathrm{mL}$ solution in chloroform. Temperature dependent UV-Vis absorption spectra were obtained using Agilent Cary 6000i UV-Vis-NIR Spectrophotometer. A Peltier temperature controller was used to regulate the temperature inside the chamber using liquid nitrogen as the coolant. Before each scan, the thin film temperature was allowed to stabilize for 2 minutes. The spectra were then used to extract Tg values in Python by the tools provided and detailed by Sam Root and colleagues. ${ }^{8}$ For comparison, oscillatory shear rheometry ${ }^{9}$ was utilized for P1 as previously reported.
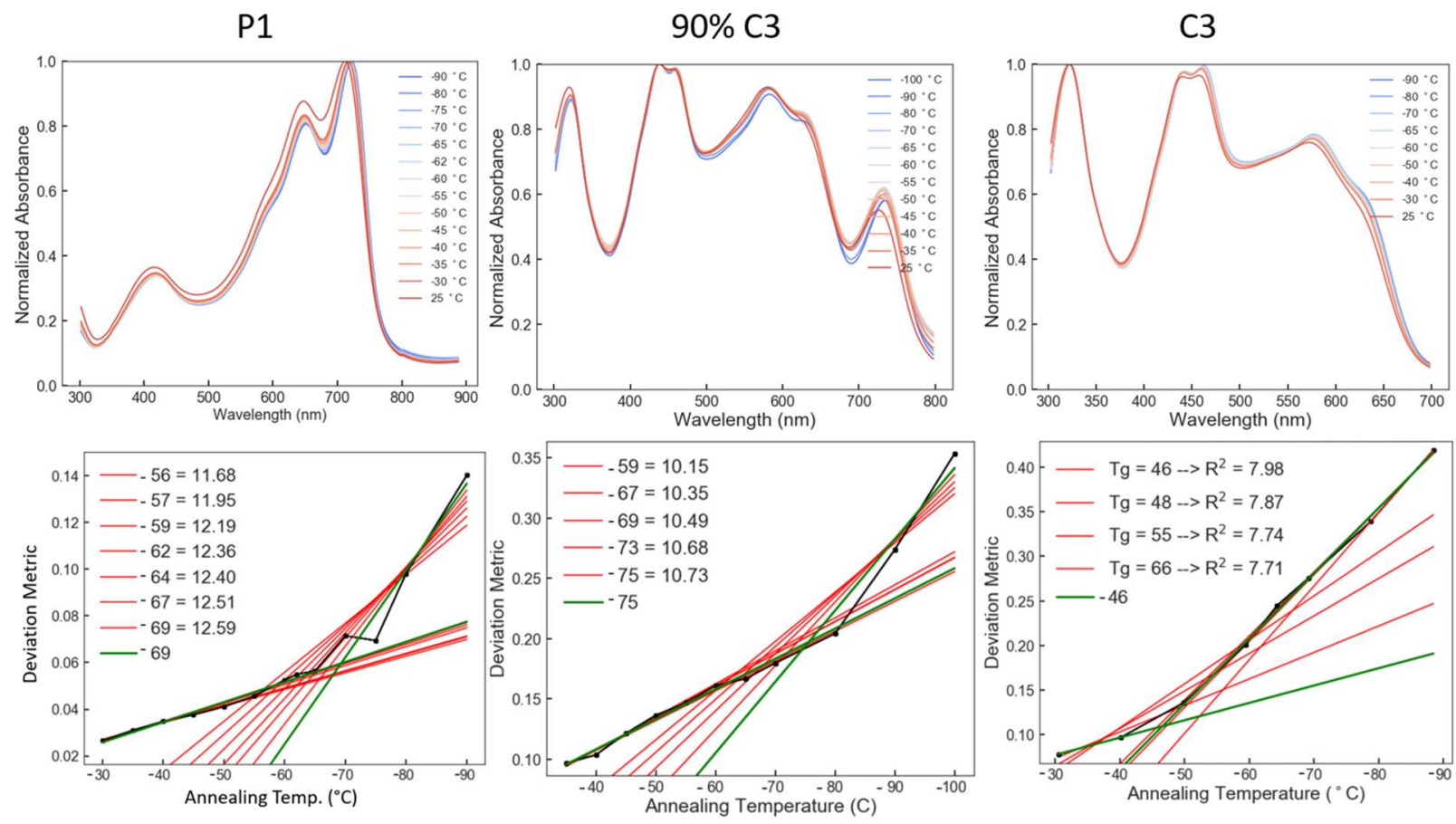

Figure S5. Temperature-dependent thin-film UV-Vis spectra used for Tg calculation and $\mathrm{Tg}$ extraction by double-linear regression of the deviation metric. 

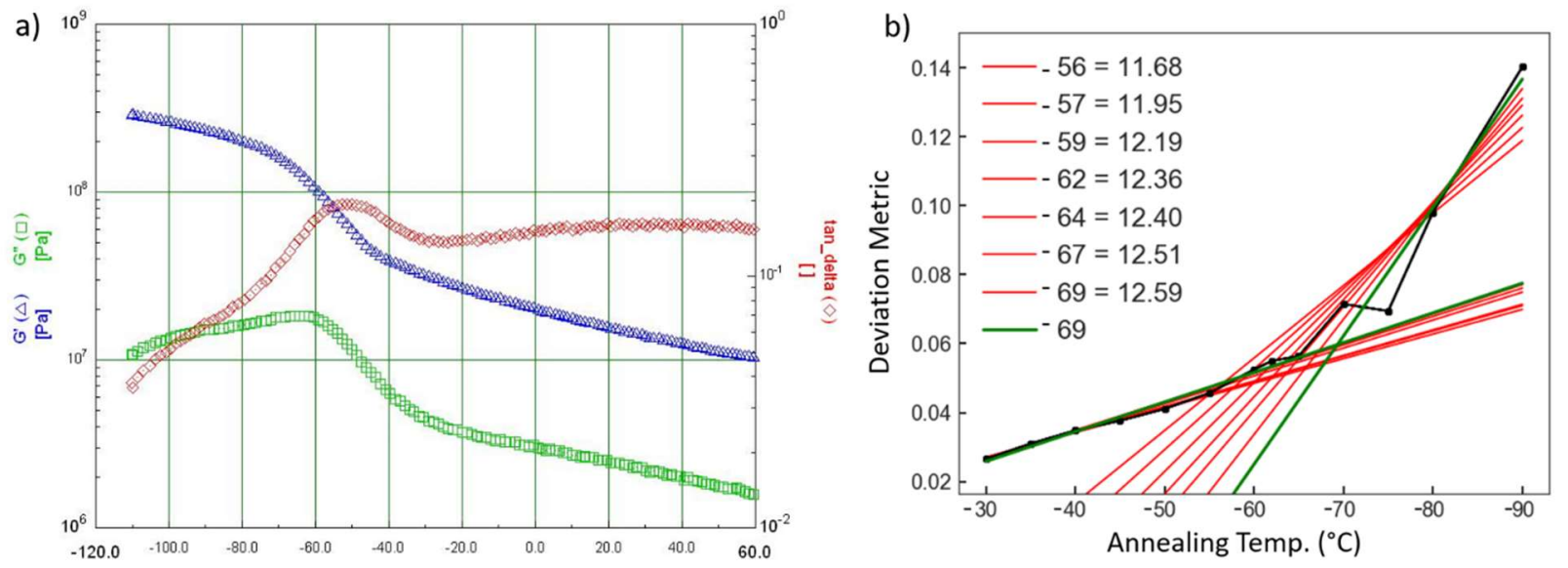

Figure S6. Extraction of Tg of polymer P1 using a) rheology, b) UV-Vis absorption method. The change in molecular segmental motion can be observed when the polymer material is cooled to as low as $-60^{\circ} \mathrm{C}$. 


\section{Mechanical Properties Measured by Buckling Method.}

All polymers were dissolved in the chloroform with the concentration of $10 \mathrm{mg} / \mathrm{mL}$. The solutions were stirred at $50{ }^{\circ} \mathrm{C}$ overnight to completely dissolve the materials. The solutions of the blends were prepared by mixing the solution of $\mathrm{P} 1$ and the matrices with different ratios. PDMS was prepared by mixing the base and the curing agent at a ratio of 10:1 (by mass). After degassing in the vacuum, the PDMS was heated to $60{ }^{\circ} \mathrm{C}$ for 2 hours. The PDMS was then cut into strips with the size of $1 \mathrm{~cm} \times 7 \mathrm{~cm} \times 2 \mathrm{~mm}$. The modulus of PDMS was measured by stretching stage (ESM303, Mark-10) and force gauge (Series 5, Mark-10) by plotting the strainstress curve. The modulus of the PDMS curing at the temperature of $60{ }^{\circ} \mathrm{C}$ for 2 hours was $\sim 1$ $\mathrm{MPa}$; after annealing the PDMS at the temperature of $140{ }^{\circ} \mathrm{C}$ for 10 minutes, the modulus increased to $\sim 1.6 \mathrm{MPa}$. The cover glass (thickness $\sim 220 \mu \mathrm{m}$ ) was cut into the size of $1 \mathrm{~cm} \times 1$ $\mathrm{cm}$. Before spin coating process $(1500 \mathrm{rpm}, 30 \mathrm{~s})$, the glass wafers were treated with uv-ozone for 30 minutes. The wafers with the semiconducting polymer layer on top were placed face down on the pre-strained (5\% strain) PDMS, which were then immersed in water. As the wafer was hydrophilic and the PDMS was hydrophobic, water would move into the interface between the polymer and the glass wafer to separate them apart. As a result, the film would be transferred onto the PDMS. The whole transfer process took about 30 minutes. The transferred film was left in the air for 2 hours for the evaporation of the water on the sample surface. After the strain applied on the PDMS was released, the buckles could form on the semiconducting polymer simultaneously. Optical microscope images were used to extract the buckles periodicity using MATLAB. The film thicknesses were measured by AFM after making a cut in different regions of the film and imaging the step edge. Film thickness of $100 \mathrm{~nm}( \pm 12.0 \mathrm{~nm})$ could be cast and transferred onto PDMS slabs. 
a)

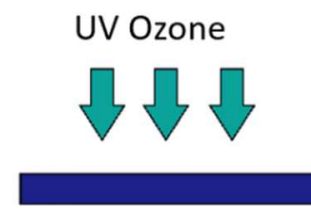

Glass wafer step 1

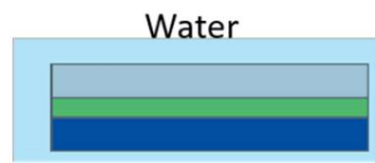

step 4
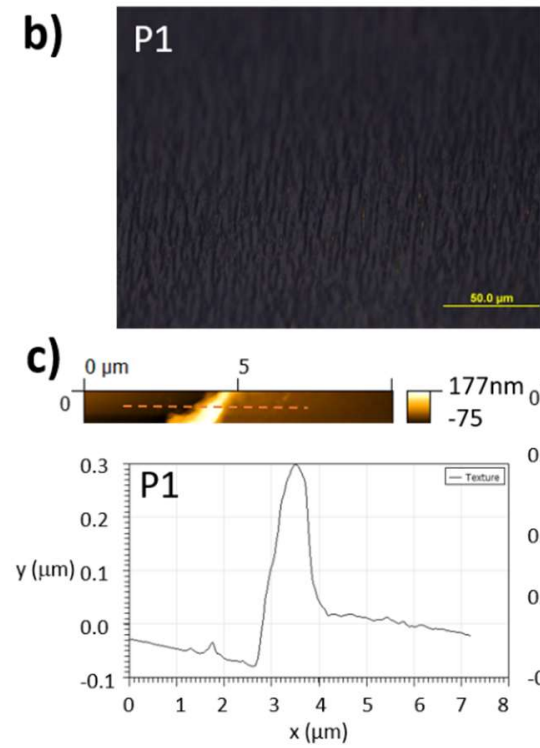

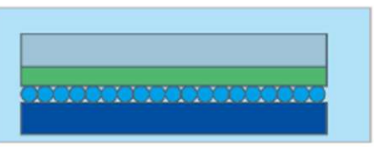

step 5
Pre-strained PDMS

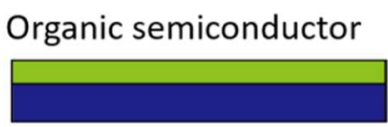

step 2

step 3

Released strain

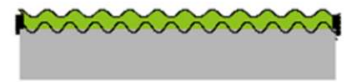

step 6
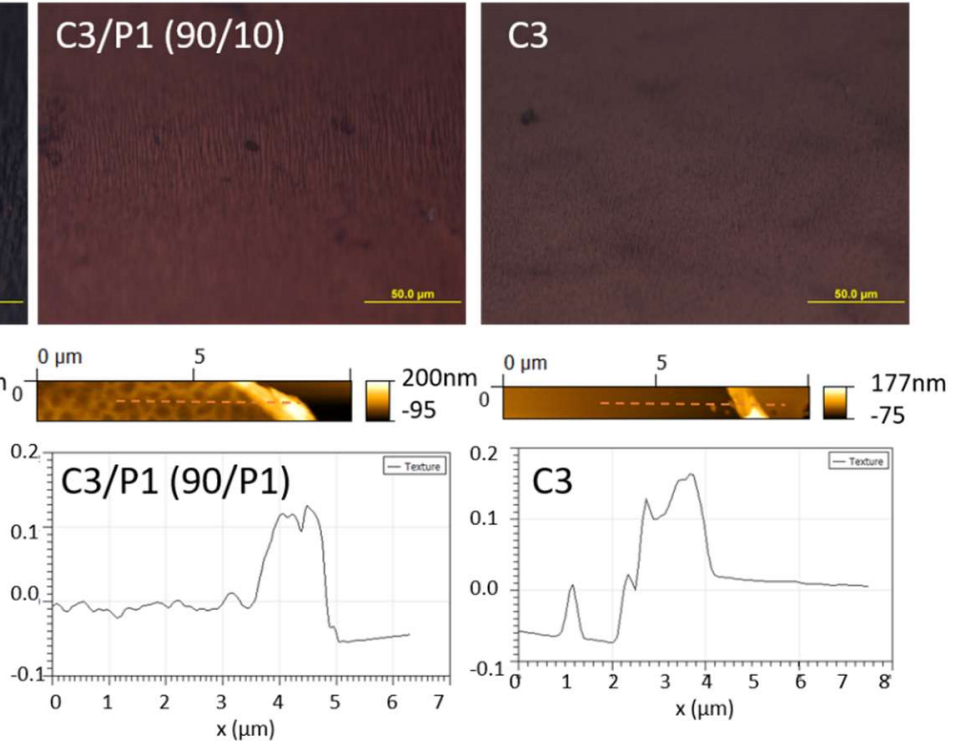

Figure S7. a) Illustration of buckling method, b) typical optical microscope images of formed buckles upon strain release and c) the AFM-extracted film thickness. 


\section{Crack-on-Set Strain Analysis and Electrical Measurement}

For the OFET devices studied under strain, the film transfer was achieved as previously described for crack-on-set analysis on PDMS slabs. The PDMS/polymer assembly was left inside a vacuum oven to remove any residual water. The dried assembly was then carefully laminated and taped onto OTS-treated substrates with the corresponding strain applied. The representative OFET hole mobilities for devices obtained by applying strain parallel to the electrodes are summarized in Table S1.
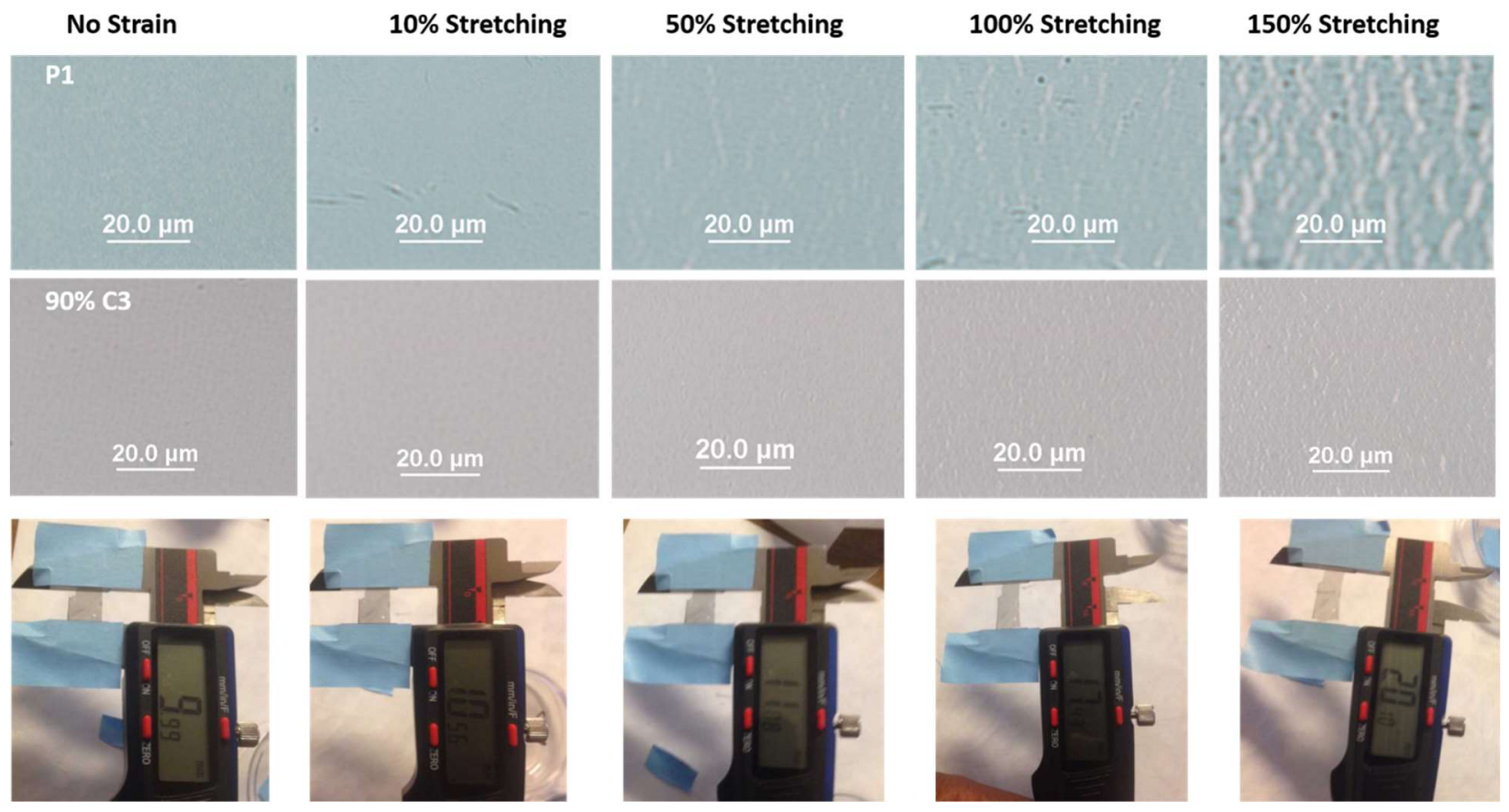

Figure S8. Crack-on-set analysis of the studied thin films on PDMS substrates. Optical microscopy was used to image the formation of microcracks in the thin films as strain was increased. The films based on nanofiber networks showed mechanical stability beyond 50\% strain. Readings on the ruler: 9.99 (no strain), 10.56 (10\% stretching), 11.08 (50\% stretching), 17.43 (100\% stretching), 20.10 (150\% stretching). 
Table S1. Summarized average hole mobilities of devices studied by parallel lamination under different strain levels.

\begin{tabular}{c|c|c|c|c}
\cline { 2 - 5 } & No strain & $\mathbf{1 0 \%}$ strain & $\mathbf{5 0 \%}$ strain & $\mathbf{1 0 0 \% \text { strain }}$ \\
\cline { 2 - 5 } & $\begin{array}{c}\boldsymbol{\mu}_{\text {max }} / \boldsymbol{\mu}_{\text {avg }} \\
\left(\mathbf{c m}^{2} \mathbf{V}^{-1} \mathbf{s}^{-1}\right)\end{array}$ & $\begin{array}{c}\boldsymbol{\mu}_{\text {max }} / \boldsymbol{\mu}_{\text {avg }} \\
\left(\mathbf{c m}^{2} \mathbf{V}^{-1} \mathbf{s}^{-1}\right)\end{array}$ & $\begin{array}{c}\boldsymbol{\mu}_{\text {max }} / \boldsymbol{\mu}_{\text {avg }} \\
\left(\mathbf{c m}^{2} \mathbf{V}^{-1} \mathbf{s}^{-1}\right)\end{array}$ & $\begin{array}{c}\boldsymbol{\mu}_{\text {max }} / \boldsymbol{\mu}_{\text {avg }} \\
\left(\mathbf{c m}^{2} \mathbf{V}^{-1} \mathbf{s}^{-1}\right)\end{array}$ \\
\hline $\mathbf{P 1}$ & $0.21 / 0.10$ & $0.15 / 0.11$ & $2.1 \mathrm{E}-3 / 5.2 \mathrm{E}-5$ & $<1 \mathrm{E}-7$ \\
\hline $\mathbf{9 0 \%} \mathbf{C 3}$ & $0.015 / 1 \mathrm{E}-3$ & $0.012 / 1.4 \mathrm{E}-3$ & $4.5 \mathrm{E}-3 / 3.3 \mathrm{E}-4$ & $1.7 \mathrm{E}-4 / 3.1 \mathrm{E}-5$ \\
\hline $\mathbf{C 3}$ & $2.0 \mathrm{E}-3 / 1.1 \mathrm{E}-3$ & $1.7 \mathrm{E}-3 / 5 \mathrm{E}-4$ & $8.4 \mathrm{E}-4 / 2.6 \mathrm{E}-5$ & $5.1 \mathrm{E}-6 / 6.5 \mathrm{E}-7$ \\
\hline
\end{tabular}



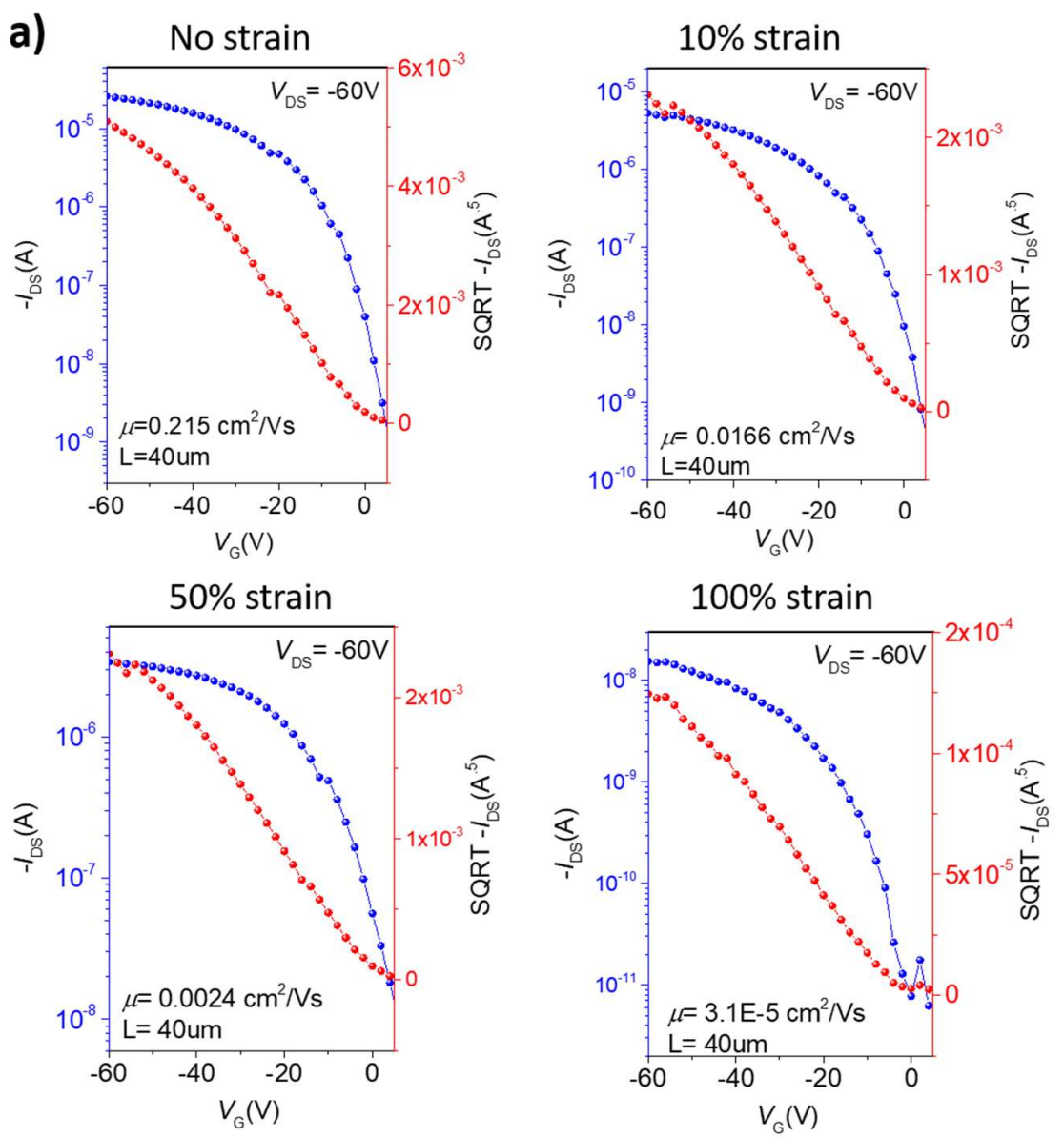

S-13 

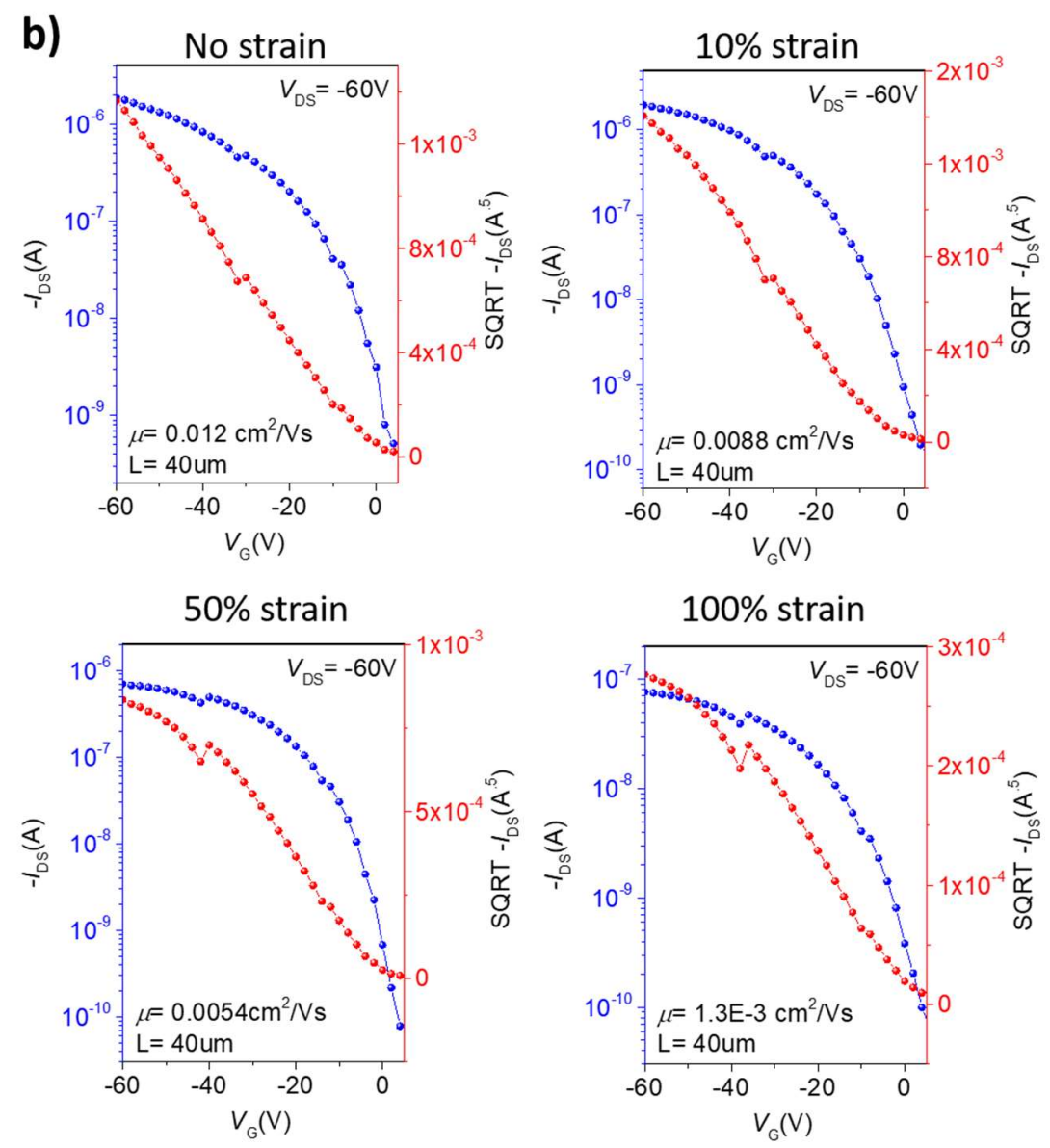

Figure S9. Representative transfer curves of representative OFET devices based on a) pure P1 and b) $90 \%$ C3 blend under various strain levels perpendicularly to the electrodes. 

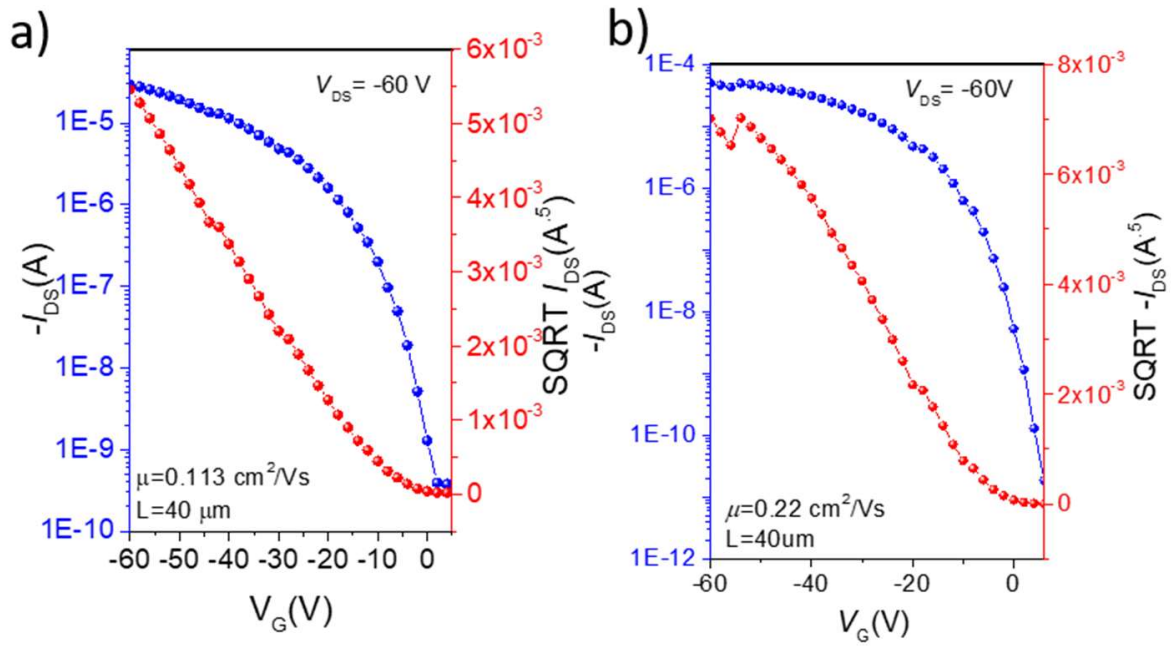

Figure S10. Representative transfer curves of the best performing OFET devices based on 90\% $\mathrm{C} 3$ blend a) before and b) after blade shearing. 


\section{REFERENCES}

1. Xue, G.; Zhao, X.; Qu, G.; Xu, T.; Gumyusenge, A.; Zhang, Z.; Zhao, Y.; Diao, Y.; Li, H.; Mei, J., Symmetry Breaking in Side Chains Leading to Mixed Orientations and Improved Charge Transport in Isoindigo-alt-Bithiophene Based Polymer Thin Films. ACS Appl. Mater. Interfaces 2017, 9, 25426-25433.

2. Mei, J.; Wu, H.-C.; Diao, Y.; Appleton, A.; Wang, H.; Zhou, Y.; Lee, W.-Y.; Kurosawa, T.; Chen, W.-C.; Bao, Z., Effect of Spacer Length of Siloxane-Terminated Side Chains on Charge Transport in Isoindigo-Based Polymer Semiconductor Thin Films. Adv. Funct. Mater. 2015, 25, 3455-3462.

3. Mei, J.; Bao, Z., Side Chain Engineering in Solution-Processable Conjugated Polymers. Chem. Mater. 2014, 26, 604-615.

4. Mei, J.; Kim, D. H.; Ayzner, A. L.; Toney, M. F.; Bao, Z., Siloxane-Terminated Solubilizing Side Chains: Bringing Conjugated Polymer Backbones Closer and Boosting Hole Mobilities in Thin-Film Transistors. J. Am. Chem. Soc. 2011, 133, 20130-20133.

5. Stalder, R.; Mei, J.; Reynolds, J. R., Isoindigo-Based Donor-Acceptor Conjugated Polymers. Macromolecules 2010, 43, 8348-8352.

6. Zhao, X.; Zhao, Y.; Ge, Q.; Butrouna, K.; Diao, Y.; Graham, K. R.; Mei, J., Complementary Semiconducting Polymer Blends: The Influence of Conjugation-Break Spacer Length in Matrix Polymers. Macromolecules 2016, 49, 2601-2608.

7. Tokarev, A.; Asheghali, D.; Griffiths, I. M.; Trotsenko, O.; Gruzd, A.; Lin, X.; Stone, H. A.; Minko, S., Touch- and Brush-Spinning of Nanofibers. Adv. Mater. 2015, 27, 6526-6532.

8. Root, S. E.; Alkhadra, M. A.; Rodriquez, D.; Printz, A. D.; Lipomi, D. J., Measuring the Glass Transition Temperature of Conjugated Polymer Films with Ultraviolet-Visible Spectroscopy. Chem. Mater. 2017, 29, 2646-2654.

9. $\quad$ Xie, R.; Lee, Y.; Aplan, M. P.; Caggiano, N. J.; Müller, C.; Colby, R. H.; Gomez, E. D., Glass Transition Temperature of Conjugated Polymers by Oscillatory Shear Rheometry. Macromolecules 2017, 50, 5146-5154. 